\title{
Predictive Factors of Social Functioning in Patients with Schizophrenia: Exploration for the Best Combination of Variables Using Data Mining
}

\author{
Sung-Man $\mathrm{Bae}^{1,2}$ \\ Seung-Hwan Lee ${ }^{1,2} \otimes$ \\ Young-Min Park ${ }^{1,2}$ \\ Myung-Ho Hyun ${ }^{3}$ \\ Hiejin Yoon ${ }^{4}$ \\ 1Department of Neuropsychiatry, \\ Ilsan Paik Hospital, \\ Inje University College of Medicine, \\ Goyang, \\ ${ }^{2}$ Clinical Emotion and \\ Cognition Research Laboratory, \\ Goyang, \\ ${ }^{3}$ Department of Psychology, \\ Chung Ang University, Seoul, Korea \\ ${ }^{4}$ Division of Child and \\ Adolescent Psychiatry, \\ LIJ Medical Center, \\ Albert Einstein College of Medicine, \\ NY, USA
}

Objective This study aimed to use data mining to explore the significantly contributing variables to good social functioning in schizophrenia patients.

Methods The study cohort comprised 67 schizophrenia patients on stable medication. A total of 51 variables ( 6 demographic data, 3 illness history, 22 social cognition, 16 neurocognition, 4 psychiatric symptoms) were input into a data-mining decision tree using the Answer Tree program to find the pathway for the best social functioning.

Results Several contributing factors for good social functioning were found. Continuous attention was the strongest contributing factor. Three variables involving best social functioning included good continuous attention, good theory of mind (TOM), and low sensitivity of disgust emotion.

Conclusion Our results confirmed the mediating roles of social cognition between neurocognition and functional outcomes, and suggested that social cognition can significantly predict social functioning in schizophrenia patients.

Psychiatry Investig 2010;7:93-101

Key Words Social functioning, Schizophrenia, Data mining, Neurocognition, Social cognition.

Received: September 7, 2009 Revised: March 9, 2010 Accepted: March 15, 2010

Available online: April 6, 2010

\section{Introduction}

Patients with schizophrenia usually have psychotic symptoms reflecting altered perception of reality such as auditory hallucination and delusion. ${ }^{1}$ These symptoms significantly prevent their interpersonal relationship, and disturb their adjustment in various social situations. ${ }^{2}$ After remission from their acute psychotic symptoms, they still have difficulty to live in the community together. One of major obstacles of schizophrenia patients that make it difficult for them to live in the community is poor social functioning. ${ }^{3}$ Particularly, chronic schizophrenia patients show impaired social problem solving, work performance, and interpersonal relationships. ${ }^{4}$

Social functioning also significantly affects the prognosis of schizophrenia patients. The relapse rate becomes higher in schizophrenia patients when they have serious functional deficit, and functional deficit has been considered as an important prognosis factor. ${ }^{4,5}$ Therefore, evaluating social functioning is important for predicting the prognosis as well as determining effective treatments for schizophrenia patients. Because the ability of social functioning can play an important role in everyday life and schizophrenia patients with low social functioning showed the poor prognosis, many researchers have focused on identifying the factors related to social functioning in these patients. ${ }^{4}$

Green ${ }^{6}$ described how specific neuropsychological defects are predictive of general functioning in schizophrenia. They found that the affected neuropsychological domains in schizo- 
phrenia patients included verbal memory, vigilance, and executive function.

Furthermore, there are reports about the relationship between neurocognitive functioning and psychotic symptoms (especially negative ones) in terms of predicting social functioning in schizophrenia patients. It was revealed that these two variables are mutually dependent and interactive.

Velligan et al. ${ }^{7}$ investigated the relationship between symptoms, cognitive function, and the ability to perform basic activities of daily living in schizophrenia, and they found that cognitive function predicted both concurrent symptoms and basic activities of daily living. Cognitive function also predicted more than $40 \%$ of the variance in scores for functional needs. However, the symptoms had little direct impact on the activities of daily living.

Recently, the social cognition has been focused on as one of the important factors influencing social functioning in schizophrenia. ${ }^{8,9}$ There is accumulating evidence of consistent relationships between social functioning and social cognition. ${ }^{10}$ Also, many researchers have found a positive relationship between neurocognition and social cognition. ${ }^{11}$ Social cognition can be defined as a 'mental process' that forms the basis of social interaction, including the ability of humans to understand intentions of other people. ${ }^{12}$ Emotional processing, social perception, social knowledge, theory of mind (TOM), and attributional bias are major domains of social cognition that are often discussed in schizophrenia research, ${ }^{8,9}$ among which emotion perception and TOM are currently receiving considerable attention. Emotional perception is mainly measured through the ability to recognize accurately the affect (emotion) of other people from facial expressions, gestures, or voices. TOM is the ability to conceptualize the mental states of others, such as having an accurate understanding of their beliefs and intentions.

While there have been vigorous efforts to explain social functioning in schizophrenia patients, only a few researches have attempted to integrate the relationships among possible factors (demographic data, neurocognition, social cognition, and psychiatric symptoms). This means that previous results may provide a limited understanding about the relationship between these factors. This prompted us to address two questions: 1) What is the best combination of variables (demographic data, illness history, neurocognition, social cognition, and psychiatric symptoms) predicting social functioning in schizophrenia? and 2) Can social cognition significantly predict social functioning in schizophrenia patients?

\section{Methods}

\section{Subjects}

Sixty seven patients with schizophrenia (33 males and 34 females) were recruited from Psychiatry Department of Inje University Ilsan Paik Hospital, Korea. The average age of the patients was $36.32 \pm 10.03$ mean \pm standard deviation (SD) \} years (Table 1). All patients met the criteria for schizophrenia based on the Structured Clinical Interview for Diagnostic and Statistical Manual of Mental Disorder, Fourth Edition (DSMIV) Axis I Disorders. ${ }^{13}$ The exclusion criteria were any other Axis I disorder except for schizophrenia, central nervous system disease (e.g., epilepsy or cerebrovascular accident), alcohol or drug abuse, electroconvulsive therapy, mental retardation, or head injury with loss of consciousness.

All subjects were taking atypical antipsychotics (risperidone, $n=20$; olanzapine, $n=15$; quetiapine, $n=15$; aripiprazole, $\mathrm{n}=10$; and ziprasidone, $\mathrm{n}=7$ ) at an overall dosage of $457.76 \pm$ 256.37 chlorpromazine (CPZ) equivalents. They were all in a symptomatically stabilized state without any dosage changes in antipsychotic medication for at least 2 weeks. Their score on the Positive and Negative Syndrome Scale (PANSS) was $67.21 \pm 23.79$. The study was approved by a Human Subjects Review Committee of Inje University Ilsan Paik Hospital and subjects participated after signing a written voluntary informed consent form. Each test were conducted and rated by same persons who were in charge of the test.

\section{Evaluation of social cognition}

\section{Theory-of-mind test}

We used the animation test to measure TOM. ${ }^{14}$ In 12 soundless animations, a large red triangle and a small blue triangle were presented moving on a white background for 35-45 seconds on a computer screen. After a subject looked at each animation, the evaluator asked "What happened in this animation?" and wrote down the subject's answer. The animations were of three types: 1) a random animation with no interactions and containing only aimless movement, 2) a goal-directed sequence that included interaction between two objects, such as one character responding to the other character but not understanding the other character's mind, and 3) the TOM

Table 1. Demographic data of the schizophrenia patients

\begin{tabular}{lc}
\hline \multicolumn{1}{c}{ Variable } & Value (mean \pm SD) \\
\hline Age (years) & $36.32 \pm 10.03$ \\
Sex & Male: 33 , Female: 34 \\
Education (years) & $12.60 \pm 2.68$ \\
Duration of illness (months) & $99.40 \pm 106.18$ \\
Number of hospitalizations & $2.61 \pm 2.40$ \\
Chlorpromazine equivalents (mg) & $457.76 \pm 256.37$ \\
PANSS total score & $67.21 \pm 23.79$ \\
PANSS positive symptoms score & $16.33 \pm 8.08$ \\
PANSS negative symptoms score & $16.14 \pm 7.81$ \\
SPQ & $29.73 \pm 13.87$
\end{tabular}

SD: standard deviation, PANSS: Positive and Negative Syndrome Scale, SPQ: Schizotypal Personality Questionnaire 
sequence, in which one character responded to the other character's mental state. Each answer was scored as 0 , 1, or 2 according to how accurately the subjects appeared to understanding the animation's intended meaning. Cronbach's a for our study was 0.71 .

\section{Facial affect identification test}

This test has been developed and standardized for the Korean population, ${ }^{15}$ and measures the ability to recognize facial expressions on the computer screen. Subjects look at pictures of seven types of affects (happy, sad, angry, fearful, surprised, disgusted, and neutral faces) and carry out tasks related to affect discrimination and affect intensity perception. The affect discrimination task involves choosing the correct affect among seven expressions when they see one emotional facet. The affect-intensity perception task involves measuring the sensitivity to slight changes in emotion, and is quantified from 0 (no affect) to 8 (maximal affect). This study measured three variables: affect discrimination, affect intensity perception, and reaction time for affect perception. Cronbach's a for our study was 0.66 .

\section{Social behavioral sequencing test}

The social behavioral sequencing test was developed by Kwon et al. ${ }^{16}$ It consists of six actual social situations, each of which consists of nine serial behaviors. The situations are 'having lunch in fast-food restaurant, seeing a doctor in hospital, buying a pair of shoes in a department store, buying a secondhand bicycle after seeing an advertisement, sending money in a bank, and getting a phone repaired in a customer service center'. Each set of nine serial behavior cards (each card is $8 \times 2$ $\mathrm{cm}$ ) is displayed to a subject, whose task is to place them in order. There is no time limit. After the subjects have finished arranging the cards, the evaluator records the order of the arranged cards. For example, the order H-D-B-Y-G-J-M-C-K would be recorded as 'HDBYGJMCK', and the answer divided into eight pairs of consecutive cards: H-D, D-B, B-Y, Y-G, G-J, J-M, M-C, and C-K. A score of 1 is assigned for each pair in the correct order, and 0 is assigned otherwise. Therefore, the maximum score for each set is 8 . The Cronbach a was 0.87 , and the test's internal consistency is very high. ${ }^{16}$

\section{Evaluation of neurocognition}

Neurocognition tests were carried using the computerized neurocognitive function test, which has been standardized for a Korea population. We used the visual continuous performance test (vCPT: CPT hit rate, CPT fail rate, CPT false number, CPT reaction time), auditory verbal learning test (VL)(VL first recall, VL fifth recall, VL total recall, VL delay recall, VL recognition), Trail-making test A, Trail-making test B, and Wisconsin card-sorting test (WCST: WCST trial completed target, WCST total error, WCST perseverative response, WCST perseverative error, WCST nonperseverative error).

\section{Evaluation of psychiatric symptoms}

We used the Schizotypal Personality Questionnaire (SPQ), and the PANSS. The SPQ was developed by Raine ${ }^{17}$ and modified by Lee et al. ${ }^{18}$ This questionnaire paraphrases the nine diagnostic criteria of schizotypal personality disorder in DSMIII-R, and consists of the idea of reference, social anxiety, magical thinking, unusual perception, eccentric behavior, lack of close friends, odd speech, constricted affect, and suspiciousness or paranoid ideas. We used a Korean standardized version of the PANSS that was originally developed by Kay et al. ${ }^{19}$

\section{Evaluation of social functioning: social role functioning test}

We used the social role functioning test (SRFT) developed by McPheeters, ${ }^{20}$ which consists of four scales: working productivity (in the subject's main role), independent living (managing of household, eating, sleeping, and hygiene care), immediate social-network relationships (close friends, spouse, and family), and extended social-network relationships (neighborhood, community, church, clubs, agencies, and recreational activities). Each scale has seven levels of functioning, from worst functioning at level 1 to best functioning at level 7, based on living during the previous 2 months. Cronbach's a for our study was 0.85 (subscale $\mathrm{a}=0.78,0.76,0.81,0.78$ ).

\section{Data management and statistical methods}

Data mining was developed by computer scientists in the early 1990s, and involves searching large bodies of data for interesting patterns and structures. ${ }^{21,22}$ Two major applications of data mining in medicine are the mining of the medical literature and patients' databases. While traditional statistics emphasizes inference and estimations, data mining emphasizes the fulfillment of a task such as classification, estimation, or knowledge discovery. Data mining uses data from naturalistic observations and is not based on an experimental design, and hence can generate new hypotheses that independent of experimental designs. We decided to use data mining to explore the clinical pathway influencing social functioning in schizophrenia.

To identify variables that strongly predict the level of social functioning in schizophrenia, 51 independent variables were assembled into a data-mining decision tree using the Answer Tree program (version 2.1). The 51 independent variables are following (Table 2): 6 demographic data (age, sex, education, job, SES, and handedness), 3 illness history (duration of illness, number of hospitalization, and medication dosage), 22 social cognition data (TOM, 7 affect discrimination task variables, 6 affect intensity perception task variables, 7 reaction time for affect perception variables, and Social Behavioral Sequencing Test), 16 neurocognition data (4 vCPT variables, 5 VL variables, 2 Trail Making Test variables, and 5 WCST vari- 
Table 2. List of variables which were assembled into a data-mining decision tree using the Answer Tree program

\begin{tabular}{|c|c|}
\hline Category & Variable \\
\hline $\begin{array}{l}\text { Demographic } \\
\text { data } \\
(\mathrm{N}=6)\end{array}$ & $\begin{array}{l}\text { Age (years) } \\
\text { Sex } \\
\text { Education (years) } \\
\text { Job } \\
\text { Socioeconomic status } \\
\text { Handedness }\end{array}$ \\
\hline $\begin{array}{l}\text { Illness } \\
\text { history } \\
(\mathrm{N}=3)\end{array}$ & $\begin{array}{l}\text { Duration of illness (months) } \\
\text { Number of hospitalizations } \\
\text { Chlorpromazine equivalents (mg) }\end{array}$ \\
\hline $\begin{array}{l}\text { Social } \\
\text { cognition } \\
\text { data }(N=22)\end{array}$ & $\begin{array}{l}\text { TOM } \\
\text { Hit rate for affect discrimination } \\
\text { (happy, fear, disgust, angry, depressed, } \\
\text { surprised, neutral) } \\
\text { Affect intensity perception (happy, fear, } \\
\text { disgust, angry, depressed, surprised) } \\
\text { Reaction time for affect perception (happy, } \\
\text { fear, disgust, angry, depressed, surprised, } \\
\text { neutral) } \\
\text { Social Behavioral Sequencing Test }\end{array}$ \\
\hline $\begin{array}{l}\text { Neurocognition } \\
\text { data } \\
(\mathrm{N}=16)\end{array}$ & $\begin{array}{l}\text { CPT hit rate } \\
\text { CPT fail rate } \\
\text { CPT false number } \\
\text { CPT reaction time } \\
\text { VL first recall } \\
\text { VL fifth recall } \\
\text { VL total recall } \\
\text { VL delay recall } \\
\text { VL recognition } \\
\text { Trail-making test A } \\
\text { Trail-making test B } \\
\text { WCST trial completed target } \\
\text { WCST total error } \\
\text { WCST perseverative response } \\
\text { WCST perseverative error } \\
\text { WCST nonperseverative error }\end{array}$ \\
\hline $\begin{array}{l}\text { Psychiatric } \\
\text { symptom } \\
(\mathrm{N}=4)\end{array}$ & $\begin{array}{l}\text { PANSS total score } \\
\text { PANSS positive symptoms score } \\
\text { PANSS negative symptoms score } \\
\text { SPQ }\end{array}$ \\
\hline
\end{tabular}

TOM: theory of mind, CPT: Continuous Performance Test, VL: verbal learning; WCST: Wisconsin Card Sorting Test, PANSS: Positive and Negative Syndrome Scale, SPQ: Schizotypal Personality Questionnaire

ables), 4 psychiatric symptoms (PANSS total, PANSS positive, PANSS negative, and SPQ).

Decision-tree analysis uses exploration and modeling to classify and predict according to decision rules through tabulating in a tree structure. It is a statistics technique that combines discriminate analysis and regression analysis, and uses data reduction and variable screening to select significant predictive variables that influence a target variable, segmentation that classifies measurement data into several types, and clas- sification that divides target variable into several grades. Because the main purpose of this research was to find the set of variables that are predictive of best social functioning in schizophrenia, we used the Classification and Regression Trees (CART) method ${ }^{23}$ that uses the decrease in the improvement of target variable based on the mean value of each variable as predicting variables.

The CART method can use nominal, ordinal, and continuous types of predictive and target variables, where the mean value increases and the improvement decreases as the nodes progress, because the CART uses the mean value of each node as the predictive value. A CART tree is a binary decision tree that is constructed by splitting a node into two child nodes repeatedly, beginning with the root node. By stopping rule, maximum tree depth is 4 levels, and case number of parent node and child node is 3 and 1 each. Missing data established surrogate rule according to the order that prediction agrees in particular node. The surrogate split method is otherwise used to deal with missing data in predictor variables. If missing value is existed for a case, the best surrogate split among all non missing predictors associated with surrogate splits will be used to decide which child node it should go. In this study, improvement degree explained by regression tree is analyzed by risk estimate. Finally, the predictive power and efficiency of the final model were calculated using a gain index.

\section{Results}

Figure 1 depicts the decision-making answer tree developed for social role functioning in schizophrenia patients. Our results indicated that the vCPT false number was the strongest factor for predicting social functioning. When the vCPT performance was good (vCPT false number $\leq 5.5$ ), the TOM acted as the next core mediator: a high TOM score $(>6)$ indicated good social role functioning (mean=17.97), whilst a low TOM score indicated poor social role functioning (mean=10.50). The social functioning was best when the following three variables were combined together sequentially (Figure 2): 1) good continuous attention (vCPT false number $\leq 5.5$, mean= $4.4), 2)$ higher TOM score $(>6$, mean=9.9), and 3) low sensitivity of disgust emotion (perceived disgust intensity of $\leq 3.5$, mean=5.7). In this pathway, if patients showed a high sensitivity to disgust emotion, the $\mathrm{VCPT}$ reaction time played the next most-important role.

On the other hand, when the continuous attention was poor (vCPT false number $>5.5$ ), socioeconomic status played the next most-important role. In this case, social role functioning was low (mean=9.60) if the socioeconomic level was low (SEM=low), whereas social role functioning was high (mean= 15.23 ) if the socioeconomic level was relatively high (SEM= middle). The social functioning level was worst when the following three variables were combined together sequentially 
(Figure 2): 1) poor continuous attention (vCPT false number $>5.5$ ), 2) low socioeconomic status, and 3) low TOM score $(\leq 3)$. In this pathway, if TOM was good (TOM score $>3$ ), social role functioning level was higher (mean=10.75). The PANSS negative symptoms score played the next most-important mediating role. Age played a mediating role when the patient showed a poor vCPT and had a high socioeconomic level (SEM $=$ middle). Social role functioning was poor (mean=13.50) in older patients (age $>41.5$ years). The duration of illness played the next most-important mediating role. But when patients were young, a lower antipsychotic dosage ( $\leq 800 \mathrm{CPZ}$ equivalents) resulted in a high social role functioning level (mean $=16.65$ ).

When patients showed poor continuous attention, low socioeconomic status, and high TOM score, negative symptoms played a mediating role. The social role functioning level was low (mean=9.50) if the negative symptom score was high (PANSS negative symptoms score $>15.07$ ), whereas social role functioning level was relatively high (mean=12.00) if the negative symptoms score was low.

Table 3 lists the risk estimate and standard error of the final model formed automatically according to the stopping rule to forecast social role functioning in schizophrenia patients. The risk estimate refers to the improvement within a group in the

Table 3. Risk table of social role functioning for the training sample in schizophrenia patients

\begin{tabular}{lc}
\hline \multicolumn{2}{c}{ Training sample } \\
\hline Risk estimate & 6.596 \\
Standard error of risk estimate & 1.566 \\
\hline
\end{tabular}

Table 4. Gain table of index values for particular nodes for predicting the social role functioning in schizophrenia

\begin{tabular}{ccccc}
\hline \multicolumn{5}{c}{ Gain Index of a particular node } \\
\hline $\begin{array}{c}\text { Node } \\
\text { number }\end{array}$ & $\begin{array}{c}\text { Data } \\
\text { points (N) }\end{array}$ & Node (\%) & Gain & Index (\%) \\
\hline 7 & 1 & 1.56 & 26.00 & 156.11 \\
14 & 1 & 1.56 & 24.00 & 144.10 \\
13 & 45 & 70.31 & 17.66 & 106.03 \\
16 & 5 & 7.81 & 16.65 & 99.99 \\
15 & 1 & 1.56 & 15.00 & 90.06 \\
18 & 3 & 4.69 & 14.33 & 86.06 \\
19 & 2 & 3.13 & 12.00 & 72.05 \\
17 & 1 & 1.56 & 11.00 & 66.05 \\
3 & 2 & 3.13 & 10.50 & 63.04 \\
20 & 2 & 3.13 & 9.50 & 57.04 \\
11 & 1 & 1.56 & 5.00 & 30.02 \\
\hline
\end{tabular}

Node number: node number in the decision-making answer tree, Data points $(\mathrm{N})$ : number of data points of a particular node, Node $(\%)$ : percentage of a particular node, Gain: predictive value of social role functioning, Index (\%): efficacy of decision-making child node, and was calculated as the total improvement minus the improvement of each node. In this case the risk estimate was 6.596 . The improvement ratio, which only applies if the root node existed and after the regression tree was formed, was 6.596/15.891. This means that 58.5\% (i.e., 100-41.5\%) more of the variance can be explained when the regression tree contains more than only a root tree.

The final results can be interpreted using a gain index produced by the gain table presented in Table 4 . This index quantifies the efficiency of estimates in each node, and can be interpreted as the production efficiency of an enterprise. In the present context, the index indicates that the decision-making for forecasting the social role functioning level in schizophrenia patients is most efficient and correct when features of seven nodes are used.

\section{Discussion}

This study examined the most suitable and efficient model for predicting the social role functioning in schizophrenia patients. The study also explored the hypothesis that social cognition may be important factor influencing social functioning in schizophrenia. One of the major findings of our study was the major contributions to predicting social role functioning made by continuous attention (by vCPT false number), TOM, perceived disgust intensity, age, $\mathrm{vCPT}$ reaction time, medication dose, illness duration, socioeconomic status, and negative symptoms (Figure 1). Among those variables, continuous attention (by the vCPT false number) was the strongest factor for predicting social functioning of schizophrenia. This means that neurocognitive functioning (e.g., continuous attention) contributes significantly to good social functioning. Particularly our results suggest that the best social functioning can be predicted when three variables are combined together sequentially: good continuous attention (by vCPT false number), good TOM performance, and low sensitivity of negative emotion (disgust) (Figure 2, upper pathway). This finding is in line with a previous one that social functioning in schizophrenia can be influenced by neurocognitive functioning, social cognition ability, symptoms, insight, and type of medication. ${ }^{24}$

Only a few researches have attempted to integrate the relationships among all possible factors (demographic characteristics, neurocognition, social cognition, and psychiatric symptoms). Pinkham and Penn ${ }^{25}$ have examined the relationships between neurocognition, social cognition, and interpersonal skill in 49 outpatients with schizophrenia and 44 non clinical participants. Results indicated that social cognition (emotion perception, social knowledge, TOM) significantly influenced to interpersonal skill beyond that of neurocognition in schizophrenia groups. But this patterns was not observed in the non clinical group.

It is generally accepted that schizophrenia patients exhibit 


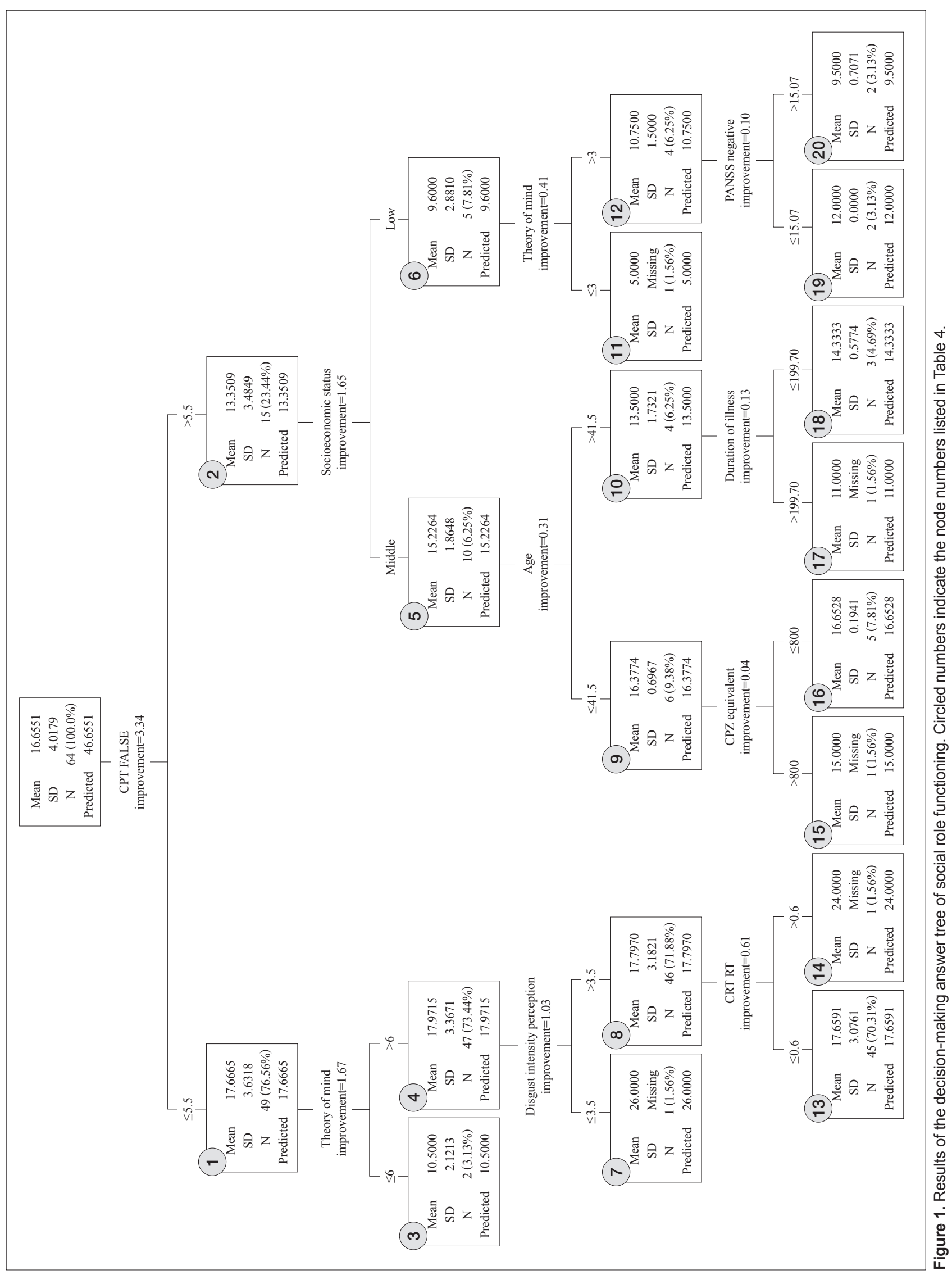


Figure 2. Pathways of the best and worst social role functioning in schizophrenia patients, showing that social cognition mediates between neurocognition and functional outcomes.

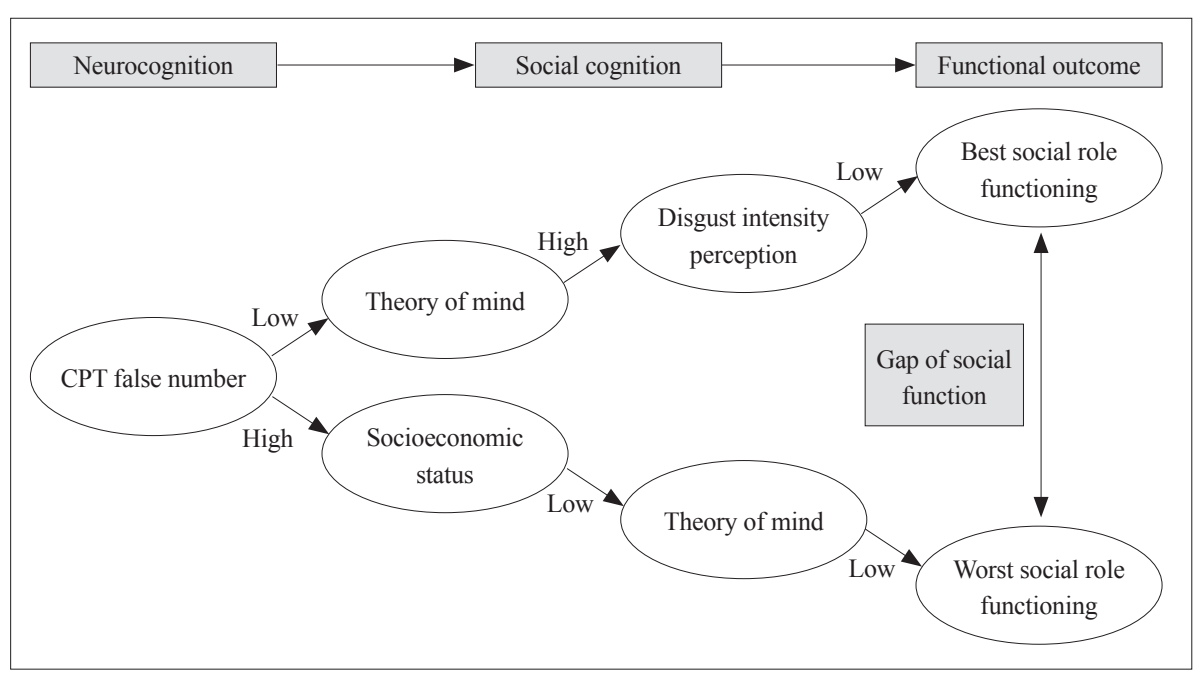

deficient attention, information processing, memory learning, and executive function relative to healthy people. Particularly, selective and continuous attention deficits in schizophrenia patients are major obstacles to achieving good social behavior. ${ }^{26}$ Continuous attention, is the strongest variable predicting social functioning of schizophrenia; verbal ability, facial emotion recognition, and social behavioral sequencing were next in explanatory powers.

Also, it has been revealed that social cognition is predictive of social functioning level in schizophrenia patients, with a TOM deficit contributing greatly to impaired social functioning in schizophrenia patients. ${ }^{27,28}$ Furthermore, Bora et al. ${ }^{29}$ investigated the relationship between TOM deficits and social functioning among 50 outpatients with schizophrenia, and found that patients with better social functioning showed significantly better performance in TOM and working memory.

Interestingly, the results of our study suggest that sensitivity to the disgust emotion is significant predictive of social role functioning. There could be several possible reasons for this unexpected result. The negative emotions of sadness, anger, and disgust cannot be classified simply or independently since they are secondary emotions expressed in everyday life that are mixed with multiple other emotions. Particularly the disgust is complex and ambiguous emotion. Even healthy subjects might have difficulty recognizing such complex emotions. Furthermore, Monkul et al. ${ }^{30}$ compared the ability to judge emotion intensity with or without context between 30 outpatients with schizophrenia and 30 demographically matched healthy subjects, and found that schizophrenia patients tend to judge anger and disgust intensities higher than healthy subjects in a contextfree condition. Moreover, the Global Assessment of Functioning scores in schizophrenia patients were significantly negatively correlated with judgments of anger $(r=-0.51)$ and disgust $(r=-0.44)$ intensities. This means that lower ratings of disgust and anger intensities are associated with better social functioning. Furthermore these results suggest that the perceived inten- sity of secondary emotion (disgust, anger) might significantly affect the social functioning level in schizophrenia patients.

On the other hand, we found that poor social role functioning can be predicted by poor continuous attention, low socioeconomic status, and poor TOM ability (Figure 2, lower pathway). This result suggests that low socioeconomic status and TOM deficit could lead the decline of social role functioning in schizophrenia patients when a large attention defect is present first. ${ }^{27,28}$ These findings support the previous assumption ${ }^{31}$ that social-cognition variables such as emotional perception, social schema, insight, and coping strategy can mediate between neurocognition functioning and social role functioning in schizophrenia. ${ }^{31}$ Sergi et al. ${ }^{32}$ used structural equation modeling to test the potential of social perception as a mediator of relations between early visual processing and functional status in schizophrenia. The mediation model indicated that early visual processing (by computerized visual masking procedures) is linked to functional status (work functioning, independent living, social functioning) through social perception (by percent of scenes correctly labeled). These findings suggest that social cognition mediates the influence of neurocognition on social role functioning in schizophrenia. Furthermore eliminating the risk factors that worsen social functioning would be an effective treatment for schizophrenia patients, with moderating risk factors by improving protective factors also being effective. $^{33}$

This study also showed that age, duration of illness, and negative symptoms significantly affect social role functioning. Social role functioning in schizophrenia was impaired in older patients (age $>41.5$ years) with a long illness (duration $>199.70$ months) and large negative symptoms score (PANSS negative symptoms score $>15.07$ ). In particular, many researches have indicated that negative symptoms are related to social functioning in schizophrenia patients. Kiang et al. ${ }^{34}$ evaluated apathy in schizophrenia and its relation to social functioning, and found that schizophrenia patients showed higher ap- 
athy than healthy control subjects, with the apathy score being correlated strongly with social functioning rather than with other negative symptoms or positive symptoms. Our study supports the hypothesis that negative symptoms such as avolition, apathy, and disinterest can explain the social functioning level in schizophrenia, including their work performance and prognosis. $^{35}$

This study was subject to several limitations. First, its design was cross-sectional, and hence long-term prospective studies are needed to evaluate the prognosis and predict social functioning in schizophrenia. Second, the tests used in this study to measure TOM are not well validated in schizophrenia patients. The TOM used in our study was originally developed for autistic children. ${ }^{36}$ Lee et al. ${ }^{37}$ reported significant TOM differences between schizophrenia patients, first-degree relatives, and healthy controls, but more studies need to apply this test to schizophrenia patients. Third, there was a statistical limitation in this study. The advantage of using a decision tree is that it can include all possible interactions in a parametric model. However, the decision tree takes a continuous variable as a noncontinuous value, and so estimation errors can occur at the boundaries where data are divided. Network analysis could be used to overcome this shortcoming. ${ }^{38}$ In addition, the present analysis have used 64 subjects and 53 variables. This can raises question of whether this is too many variables and whether just about any classification or discrimination technique is going to find a very good solution (possibly a very samplespecific solution) when using so many variables to separate so few subjects. But CART algorithm is especially adept at detecting relevant interaction between variables, ${ }^{39}$ and has used in a few similar condition to our study. ${ }^{39-41}$

Nevertheless, we have tried to determine the best model for predicting the best social role functioning in schizophrenia. And, our study suggest that combination of three variables including good continuous attention (by vCPT false number), good TOM performance, and low sensitivity of disgust emotion might be predict the best social role functioning in schizophrenia. These results may contribute to predicting exact social function, prognosis of schizophrenia, and establishing an effective remediation treatment plan. And these results also suggest social cognition can significantly predict social functioning in schizophrenia patients.

\section{-Acknowledgments}

This work was supported by a grant from the Korea Science and Engineering Foundation (KOSEF) funded by the Korean government (MOST) (No. M10644000005-06N4400-00510).

\section{REFERENCES}

1. Andreasen NC. Positive vs. negative schizophrenia: a critical evaluation. Schizophr Bull 1985;11:380-389.

2. Brekke J, Kay DD, Lee KS, Green MF. Biosocial pathways to functional outcome in schizophrenia. Schizophr Res 2005;80:213-225.
3. Lee JI, Kim JN, Kim H, Park YM, Lee SH. Impairment of theory of mind in patients with schizophrenia and their first degree relatives. In: Proceedings of the Korean Academy of Schizophrenia Autumn Academic Meeting. Seoul: Jungang Moonhwa, 2006. p.90.

4. Kim SJ, Han YS. The relation of social function, cognitive function and symptom of chronic schizophrenia. Korean J Clin Psychol 1997; 16:27-40.

5. Couture SM, Penn DL, Roberts DL. The functional significance of social cognition in schizophrenia: a review. Schizophr Bull 2006;32 suppl $1: \mathrm{S} 44-\mathrm{S} 63$.

6. Green MF. What are the functional consequences of neurocognitive deficits in schizophrenia? Am J Psychiatry 1996;153:321-330.

7. Velligan DI, Mahurin RK, Diamond PL, Hazleton BC, Eckert SL, Miller AL. The functional significance of symptomatology and cognitive function in schizophrenia. Schizophr Res 1997;25:21-31.

8. Green MF, Olivier B, Crawley JN, Penn DL, Silverstein S. Social cognition in schizophrenia: recommendations from the measurement and tr-eatment research to improve cognition in schizophrenia new approaches conference. Schizophr Bull 2005;31:882-887.

9. Green MF, Penn DL, Bentall R, Carpenter WT, Gaebel W, Gur RC, et al. Social cognition in schizophrenia: an NIMH workshop on definitions, assessment, and research opportunities. Schizophr Bull 2008;34: 1211-1220.

10. Hooker C, Park S. Emotion processing and its relationship to social functioning in schizophrenia patients. Psychiatry Res 2002;112:41-50.

11. Kee KS, Kern RS, Green MF. Perception and emotion and neurocognitive functioning in schizophrenia: what's the link? Psychiatry Res 1998;81:57-65.

12. Cacioppo JT, Berntson GG, Adolphs R, Carter CS, Davidson RI, McClintock MK, et al. Foundations in Social Neuroscience. Cambridge, MA: MIT press, 2002, p.367-368

13. Frith CD, Corcoran R. Exploring 'theory of mind' in people with schizophrenia. Psychol Med 1996;26:521-530.

14. Abell F, Happé F, Frith U. Do triangles play tricks? Attribution of mental states to animated shapes in normal and abnormal development. Cogn Dev 2000;15:1-16.

15. Lee WH, Chae JH, Bahk WM, Lee KU. Development and its preliminary standardization of pictures of facial expressions for affective neu-rosciences. J Korean Neuropsychiatr Assoc 2004;43:552-558.

16. Kwon JH, Chun JS, Choi KH. Validation study of the Social Behavior Sequencing Task: the assessment of the social-cognitive ability of schizophrenics. Korean J Clin Psychol 2003;22:629-630.

17. Raine A. The SPQ: a scale for the assessment of schizotypal personality based on DSM-III-R criteria. Schizophr Bull 1991;17:555-564.

18. Lee SH, Chung YC, Nam M, Lee KJ, Kim H, Bae SM, et al. A confirmatory factor analysis of Schizotypal Personality Questionnaire (SPQ) in Korean college students. Korean J Clin Psychol 2005;24: 663-675.

19. Kay SR, Fiszbein A, Opler LA. The positive and negative syndrome scale (PANSS) for schizophrenia. Schizophr Bull 1987;13:261-276.

20. McPheeters HL. Statewide mental health outcome evaluation: a perspective of two southern states. Community Ment Health J 1984;20: 44-55.

21. Baca-García E, Perez-Rodriguez MM, Basurte-Villamor I, Saiz-Ruiz J, Leiva-Murillo JM, de Prado-Cumplido M, et al. Using data mining to explore complex clinical decisions: a study of hospitalization after a suicide attempt. J Clin Psychiatry 2006;67:1124-1132.

22. Hand DJ. Mining medical data. Stat Methods Med Res 2000;9:305307.

23. Breiman L, Friedman JH, Olshen RA, Stone CJ. Classification and regression trees. Belmont: Wadsworth; 1984.

24. Brekke JS, Hoe M, Long J, Green MF. How neurocognition and social cognition influence functional change during community-based psychosocial rehabilitation for individuals with schizophrenia. Schizophr Bull 2007;33:1247-1256. 
25. Pinkham AE, Penn DL. Neurocognitive and social cognitive predictors of interpersonal skill in schizophrenia. Psychiatry Res 2006;143: 167-178.

26. Combs DR, Gouvier WD. The role of attention in affect perception: an examination of Mirsky's four factor model of attention in chronic schizophrenia. Schizophr Bull 2004;30:727-738.

27. Brüne M. "Theory of mind" in schizophrenia: a review of the literature. Schizophr Bull 2005;31:21-42.

28. Jahshan CS, Sergi MJ. Theory of mind, neurocognition, and functional status in schizotypy. Schizophr Res 2007;89:278-286.

29. Bora E, Eryavuz A, Kayahan B, Sungu G, Veznedaroglu B. Social functioning, theory of mind and neurocognition in outpatients with schizophrenia; mental state decoding may be a better predictor of social functioning than mental state reasoning. Psychiatry Res 2006; 145:95-103.

30. Monkul ES, Green MJ, Barrett JA, Robinson JL, Velligan DI, Glahn DC. A social cognitive approach to emotional intensity judgment deficits in schizophrenia. Schizophr Res 2007;94:245-252.

31. Green MF, Nuechterlein KH. Should schizophrenia be treated as a neurocognitive disorder? Schizophr Bull 1999;25:309-319.

32. Sergi MJ, Rassovsky Y, Nuechterlein KH, Green MF. Social perception as a mediator of the influence of early visual processing on functional status in schizophrenia. Am J Psychiatry 2006;163:448-454.

33. Hawkins JD, Graham JW, Maguin E, Abbot R, Hill KG, Catalano RF. Exploring the effects of age of alcohol use initiation and psychosocial risk factors on subsequent alcohol misuse. J Stud Alcohol 1997; $58: 280-290$
34. Kiang M, Christensen BK, Remington G, Kapur S. Apathy in schizophrenia: clinical correlates and association with functional outcome. Schizophr Res 2003;63:79-88.

35. Kurtz MM, Moberg PJ, Ragland JD, Gur RC, Gur RE. Symptoms versus neurocognitive test performance as predictors of psychosocial status in schizophrenia: a 1- and 4-year prospective study. Schizophr Bull 2005;31:167-174.

36. Pickup GJ, Frith CD. Theory of mind impairments in schizophrenia: symptomatology, severity and specificity. Psychol Med 2001;31:207220.

37. Lee SH, Jung HT, Lee J. Concept and neurobiology of social cognition in schizophrenia. J Korean Neuropsychiatr Assoc 2006;45:183190.

38. Newman ME. Modularity and community structure in networks. Proc Natl Acad Sci U S A 2006;103:8577-8582.

39. Augustin S, Muntaner L, Altamirano JT, González A, Saperas E, Dot $\mathrm{J}$, et al. Predicting early mortality after acute variceal hemorrhage based on classification and regression tree analysis. Clin Gastroenterol Hepatol 2009;7:1347-1354.

40. Lossos IS, Breuer R, Intrator O, Lossos A. Cerebrospinal fluid lactate dehydrogenase isoenzyme analysis for the diagnosis of central nervous system involvement in hematooncologic patients. Cancer 2000;88: 1599-1604.

41. Lysaker PH, Lancaster RS, Nees MA, Davis LW. Attributional style and symptoms as predictors of social function in schizophrenia. $\mathrm{J}$ Rehabil Res Dev 2004;41:225-232. 